\title{
Pattern and Dynamics of Distant Metastases in Metastatic Colorectal Cancer
}

\author{
Julian Walter Holch ${ }^{a, b}$ Maximilian Demmer ${ }^{a} \quad$ Charlotte Lamersdorfa Marlies Michla, b \\ Christoph Schulz ${ }^{a, b}$ Jobst Christian von Einem ${ }^{a, b}$ Dominik Paul Modest ${ }^{a, b}$ \\ Volker Heinemann ${ }^{a}$ b \\ a Department of Medical Oncology, Comprehensive Cancer Center Munich, University Hospital Grosshadern, Ludwig-Maximilians- \\ University Munich, Munich, Germany;

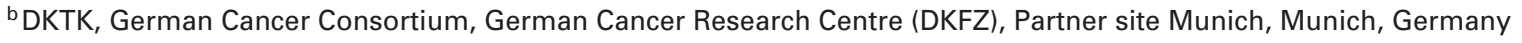

\section{Keywords}

Metastatic colorectal cancer · Localization · Incidence . Development of metastases - Metachronous metastases . Synchronous metastases

\section{Summary}

Background: Few studies report the incidence of metastatic patterns in colorectal cancer. Furthermore, little is known about dynamic aspects of these metastases during the course of disease. Methods: This retrospective cohort study involved 385 patients who received anti-tumor treatment at our institution (Department of Medical Oncology, University Hospital Grosshadern, LudwigMaximilians-University Munich, Germany) for metastatic colorectal adenocarcinoma between 2007 and 2014. We reviewed all available imaging results of these patients to document the presence and detailed localization of metastases. Results: Most of the evaluated patients were initially diagnosed with metastases in the liver (70\%), followed by the lungs (24\%), distant lymph nodes (16\%), and peritoneum $(15 \%)$, besides rare anatomical sites $(<5 \%)$. Colon and rectal cancer as well as synchronous and metachronous metastases differed with regard to the pattern of individual metastatic sites. The median time to first progressive disease (PD) with new metastases was 12.6 months. The time intervals between first and second as well as second and third PD with new metastases were comparable with 10.5 and 10.8 months, respectively. At initial diagnosis, the mean number of metastatic sites was 1.4 and increased to 2.6 at the third PD with new metastases. For patients with initially one metastatic site, the mean number increased to 2.2. Con- clusion: The present analysis provides detailed information on the pattern and evolution of colorectal cancer metastases over time. Thus, it may establish the basis for prospective future research in this field.

(C) 2017 S. Karger GmbH, Freiburg

\section{Introduction}

Colorectal cancer (CRC) is the third most frequent type of cancer in Germany with expected 61,000 newly diagnosed cases in 2016 [1]. About half of all CRC patients develop distant metastases [2-4]. Most of them finally succumb to the metastatic disease [1]. Approximately $25 \%$ of the patients are initially diagnosed in the metastatic stage (synchronous metastases) and 25\% develop metastatic spread during follow-up (metachronous metastases) [2, 4-6].

Survival differences were found for these two groups, with synchronous metastases considered to be of worse prognostic value compared to metachronous metastases [7]. Furthermore, the localization of metastases (e.g. peritoneal or distant lymph node metastases) and the number of metastatic sites have been reported to be of prognostic value [8-10].

Regarding the prognostic implications of specific metastatic patterns, only few epidemiological studies analyzing their incidence are available [6]. Moreover, information on occurrence, localization, and number of metastatic sites is not standardized in the reporting of clinical trials and is therefore not reported consistently [11]. Besides the localization and pattern of colorectal cancer metastases, only little is known about dynamic aspects regarding the development of new metastases during the course of disease.

\section{KARGER}

(c) 2017 S. Karger GmbH, Freiburg
Dr. med. Julian W. Holch

Klinikum der Universität München - Campus Großhadern, Ludwig-Maximilians-Universität München Marchioninistraße 15, 81377 München, Germany Julian.Holch@ @ed.uni-muenchen.de 
Table 1. Demographic and baseline characteristics of the analyzed patient population

\begin{tabular}{lll}
\hline & $\%$ & $\mathrm{~N}$ \\
\hline Number of patients & 100 & 385 \\
\hline Sex & & \\
$\quad$ Male & 63.6 & 245 \\
$\quad$ Female & 36.4 & 140 \\
\hline Age, years & & \\
$\quad$ Median, range & & $61(18-86)$ \\
$\quad \leq 65$ & 66.7 & 257 \\
$>65$ & 33.2 & 128 \\
\hline
\end{tabular}

Initial stage (UICC)

\begin{tabular}{lrr} 
I & 4.9 & 19 \\
II & 9.6 & 37 \\
III & 20.3 & 78 \\
IV & 59.7 & 230 \\
n.r. & 5.5 & 21 \\
\hline Primary tumor location & & \\
Colon & 61.3 & 236 \\
Rectum & 34.5 & 133 \\
Colon and rectum & 1.0 & 4 \\
n.r. & 3.1 & 12
\end{tabular}

\begin{tabular}{lrr}
\hline Resection of the primary & & \\
$\quad$ Yes & 83.1 & 320 \\
No & 16.9 & 65 \\
\hline Development of metastases & & \\
$\quad$ Metachronous & 36.6 & 141 \\
$\quad$ Synchronous & 63.4 & 244 \\
\hline
\end{tabular}

\begin{tabular}{lrr}
\hline Histological grading & & \\
G1 & 1.8 & 7 \\
G2 & 61.3 & 236 \\
G3 & 29.9 & 115 \\
n.r. & 7.0 & 27 \\
\hline RAS mutation & & \\
Yes & 45.5 & 175 \\
No & 30.6 & 118 \\
n.r. & 23.9 & 92 \\
\hline BRAF mutation & & \\
Yes & 1.6 & 6 \\
No & 3.9 & 15 \\
n.r. & 94.5 & 364 \\
\hline
\end{tabular}

n.r. $=$ Not reported.

Hence, the present study analyzed the detailed localization and development of new colorectal cancer metastases over time in a single-center patient cohort. To the best of our knowledge, this is the first study that investigates long-term dynamic aspects of colorectal cancer metastases during the course of disease.
Table 2. Treatment for metastatic colorectal cancer in the analyzed patient population

\begin{tabular}{llc}
\hline & $\%$ & $\mathrm{~N}$ \\
\hline Number of patients & 100 & 385 \\
\hline Neoadjuvant radiochemotherapy & 17.4 & 67 \\
Yes & 82.6 & 318 \\
No & & \\
\hline Adjuvant chemotherapy & 22.6 & 87 \\
Yes & 77.4 & 298 \\
No & & \\
\hline Palliative chemotherapy & 90.1 & 347 \\
1st line & 44.3 & 154 \\
$\quad$ 1st line plus anti-VEGF & 15.8 & 55 \\
$\quad$ 1st line plus anti-EGFR & 68.6 & 264 \\
2nd line & 36.3 & 126 \\
$\quad$ 2nd line plus anti-VEGF & 17.3 & 60 \\
2nd line plus anti-EGFR & 45.2 & 174 \\
3rd line & 47.7 & 83 \\
$\quad$ 3rd line plus anti-VEGF & 23.6 & 41 \\
$\quad$ 3rd line plus anti-EGFR & & \\
\hline Local ablative therapy for metastatic disease & & 207 \\
Yes & 53.8 & 178 \\
No & 46.2 & \\
\hline
\end{tabular}

EGFR $=$ Epithelial growth factor receptor; VEGF = vascular endothelial growth factor.

\section{Patients and Methods}

\section{Patient Population}

The patient population for this observational cohort study derived from the outpatient clinic of the Department of Medical Oncology at the University Hospital Grosshadern, Ludwig-Maximilians-University (LMU) Munich, Germany. A database research was performed to identify patients who were treated for histologically proven metastatic colorectal adenocarcinoma between 2007 and 2014. Medical records were accessed for demographic factors, primary tumor location, stage of disease at first presentation (Union for International Cancer Control (UICC)), development of metastases (synchronous vs. metachronous), tumor-related characteristics (histology, RAS and BRAF mutation status) as well as information regarding antineoplastic treatment. The study received approval from the ethical committee of the LMU Munich.

\section{Evaluation of Colorectal Cancer Metastases}

All available imaging results (computed tomography and magnet resonance imaging) from each patient were reviewed to document the time of occurrence and detailed anatomical localization of metastases. Board-certified radiologists validated all imaging results of this study.

\section{Statistical Analysis}

Where indicated, median or arithmetic mean values were used to describe continuous variables. Categorical variables were expressed as totals and frequencies. The frequencies of specific colorectal cancer metastases at first diagnosis were illustrated using bar diagrams. The combination of three metastatic sites was illustrated by means of a Venn diagram. The time to development of new metastases was calculated from first or previous diagnosis of metastases to the occurrence of new metastatic lesions as reported by imaging results. Time intervals, summarized as medians, and $95 \%$ confidence intervals were derived from an inverse Kaplan-Meier plot. 


\section{Results}

\section{Patient Population}

Overall, 385 patients were identified who received anti-tumor therapy for metastatic colorectal cancer (mCRC) at the outpatient clinic of the Department of Medical Oncology at the University Hospital Grosshadern (LMU Munich), Germany, between 2007 and 2014. Demographic and baseline characteristics are shown in table 1. In table 2, details about the antineoplastic treatment of this cohort are listed.

\section{Localization of Colorectal Cancer Metastases at First Diagnosis}

We analyzed the anatomical localization of metastases for each patient of the cohort according to the baseline imaging results. Figure 1 illustrates the frequencies of specific metastases in the patient cohort. Liver metastases were present in $70 \%$ of the patients. Less frequent were pulmonary, distant lymph node, and peritoneal metastases (15-25\%). Among other anatomical sites, the bones and the central nervous system were rarely affected ( 4 and $2 \%$, respectively).

Focusing on the three most frequent metastatic sites, namely metastases in the liver, lungs, and distant lymph nodes (fig. 2), $47.5 \%$ of the patients presented with metastases confined to the liver, whereas metastases confined to the lungs or distant lymph nodes were less frequent ( 6.0 and $3.4 \%$, respectively). The combination of hepatic and pulmonary metastases was observed in $7.8 \%$ of the patients. Only a minority of patients (1.8\%) had metastases to all three sites.

\section{Localization of Colorectal Cancer Metastases at First Diagnosis according to Primary Tumor Location}

We compared the frequencies of specific metastases according to the primary tumor location (fig. 3). Both in colon and rectal cancer, the liver constituted the most frequent metastatic site, albeit the frequency in colon cancer $(71 \%)$ exceeded the one in rectal cancer $(60 \%)$. The difference was more pronounced for peritoneal involvement, which occurred six times more frequent in colon cancer. Of the patients with colon cancer, $23 \%$ were diagnosed with peritoneal carcinomatosis (vs. $4 \%$ in rectal cancer). In contrast, pulmonary metastases were about twice as frequent in rectal cancer compared to colon cancer (30 vs. 17\%).

\section{Localization of Synchronous and Metachronous Colorectal \\ Cancer Metastases}

We compared the frequencies of specific metastases according to their development, i.e. synchronous versus metachronous metastases (fig. 4). Of note, metachronous metastases were diagnosed with a median time of 20.0 months after diagnosis of CRC. Among the patients with metachronous metastases, $49 \%$ showed liver involvement, whereas this frequency was much higher in patients with synchronous metastases (82\%). Additionally, peritoneal involvement was more often observed in synchronous metastases (17 vs. $11 \%)$. In contrast, pulmonary and distant lymph node metastases were more frequent in patients with metachronous metastases ( 29 vs. $22 \%$ and 19 vs. $14 \%$, respectively).

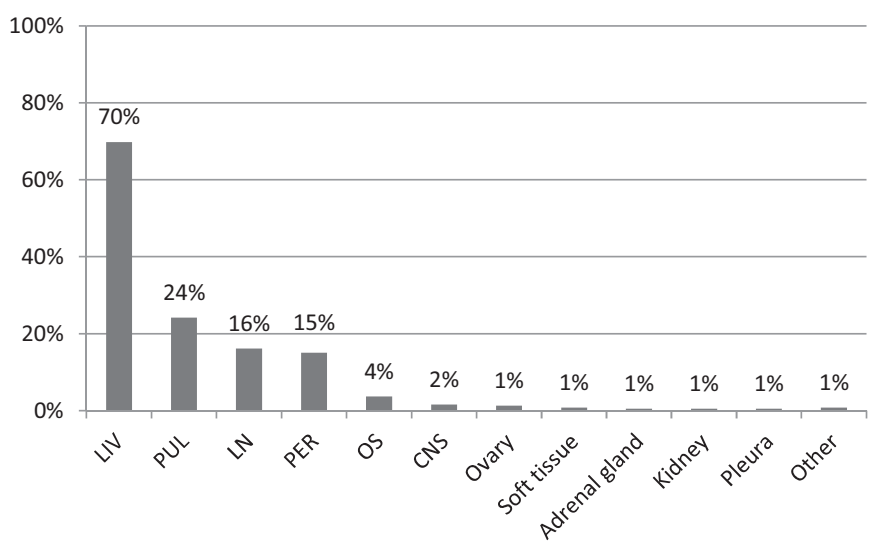

Fig. 1. Involved anatomical sites in patients with first presentation of metastatic colorectal cancer $(\mathrm{n}=385)$. LIV = Liver; PUL = lungs; $\mathrm{LN}=$ distant lymph node; $\mathrm{PER}=$ peritoneum; $\mathrm{OS}=$ bones $; \mathrm{CNS}=$ central nervous system.

Fig. 2. Combination of liver, pulmonary, and distant lymph node metastases in patients with first presentation of metastatic colorectal cancer $(\mathrm{n}=385)$. LIV = Liver; PUL = lungs; LN = distant lymph node.

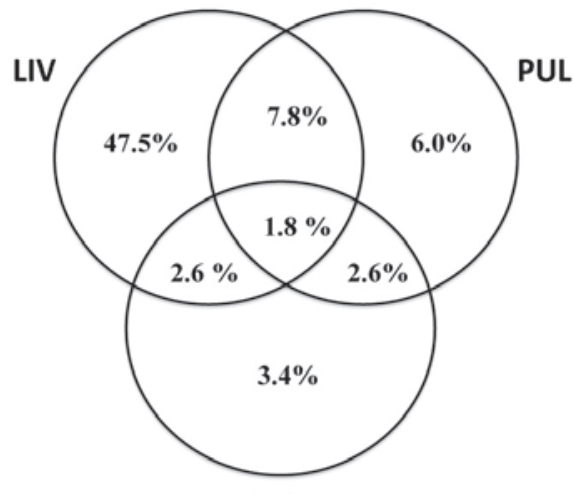

LN

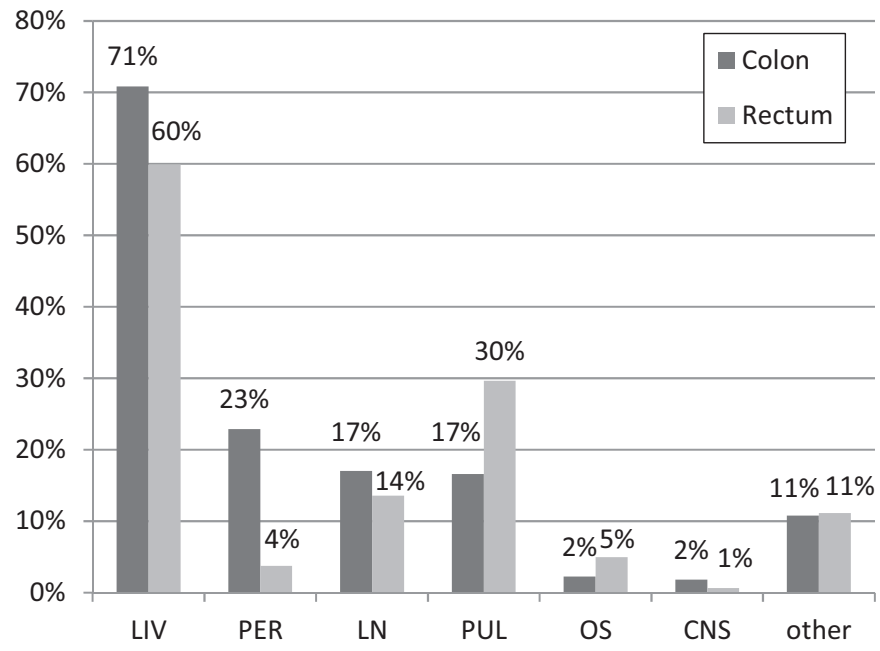

Fig. 3. Involved metastatic sites at first presentation of colon $(n=223)$ and rectal $(\mathrm{n}=162)$ cancer patients. $\mathrm{LIV}=$ Liver; $\mathrm{PER}=$ peritoneum; $\mathrm{LN}=$ distant lymph node; $\mathrm{PUL}=$ lungs; $\mathrm{OS}=$ bones; $\mathrm{CNS}=$ central nervous system.

\section{Number of Involved Metastatic Sites}

In both synchronous and metachronous metastasis, about $70 \%$ of the patients only showed one anatomical site to be affected by metastases at the time when baseline imaging was performed 
Table 3. Time to the development of further metastases in patients with metastatic colorectal cancer $(\mathrm{n}=385)$; displayed is the time from a previous diagnosis of new metastases to the development of further metastases

\begin{tabular}{llcc}
\hline & \multicolumn{2}{l}{ PD with new metastases } & \\
\cline { 2 - 4 } & first & second & third \\
\hline Time to new metastases, months & 12.6 & 10.5 & 10.8 \\
$95 \%$ confidence interval, months & $11.4-13.7$ & $8.5-12.4$ & $9.4-13.6$ \\
\hline PD = Progressive disease. & & & \\
\hline
\end{tabular}

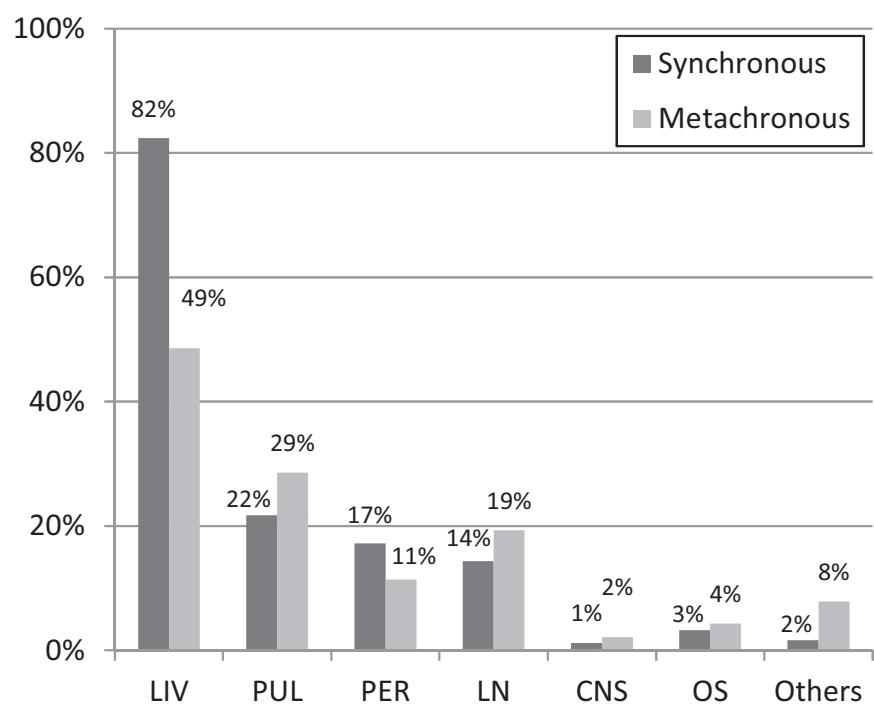

Fig. 4. Involved anatomical sites at first presentation in patients with synchronous $(\mathrm{n}=244)$ and metachronous $(\mathrm{n}=140)$ colorectal cancer metastases. LIV = Liver; PUL = lungs; PER = peritoneum; $\mathrm{LN}=$ distant lymph node, $\mathrm{CNS}=$ central nervous system; OS = bones.

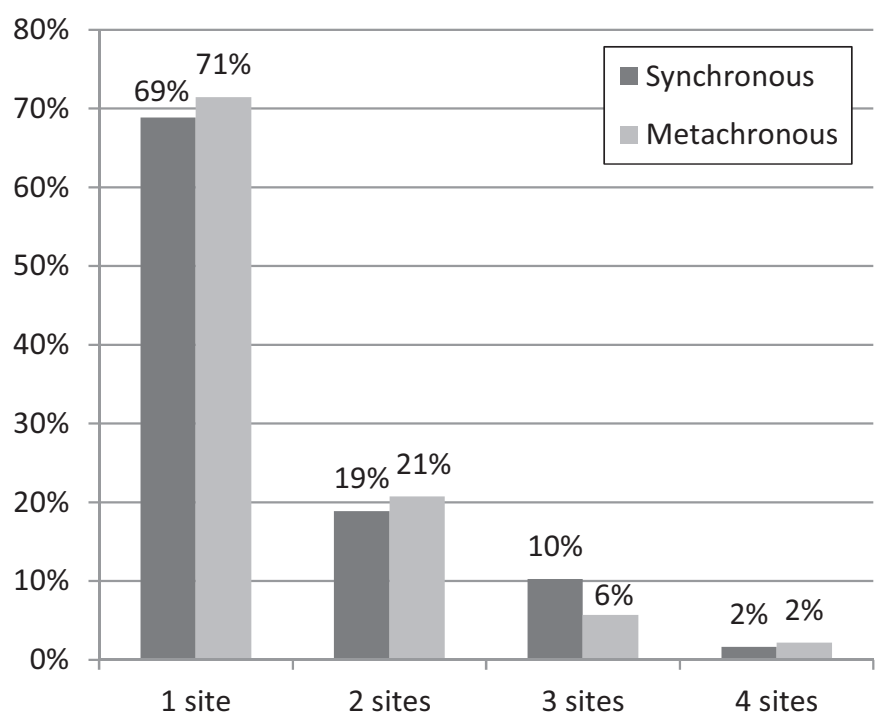

Fig. 5. Number of involved anatomical sites in patients with first presentation of metastatic colorectal cancer $(\mathrm{n}=385)$.

(fig. 5). Two anatomical sites were involved in 19 and $21 \%$ of patients with synchronous and metachronous metastases, respectively. The combination of three (10 and $6 \%$ ) or four metastatic sites (both $<5 \%$ ) at baseline were far less frequent.

\section{Dynamics of Colorectal Cancer Metastases}

We examined the time to the development of new metastases (table 3). From the first presentation of mCRC, the development of new metastatic lesions (i.e. first progressive disease (PD) with new metastases) took a median time of 12.6 months. The time to the formation of further metastases (second and third PD with new metastases) was in a comparable range with 10.5 and 10.8 months.

Finally, the number of involved metastatic sites over the course of disease was evaluated. In baseline imaging, a mean number of 1.4 sites were involved in the dissemination of colorectal cancer (fig. 6). During the course of disease, the number increased up to 2.6 at the third PD with new metastases. For patients with initially one metastatic site, the mean number at the third PD was lower with 2.2 affected sites.

\section{Discussion}

Up to date, few data exist on the organ-specific incidence of colorectal cancer metastases. Furthermore, little is known about the dynamics of the metastases during the course of disease. To the best of our knowledge, the present analysis is the first to evaluate the detailed localization of colorectal cancer metastases along with dynamic aspects.

We observed a mean number of 1.4 metastatic sites at first diagnosis in the evaluated patients. Over the course of the disease, the number increased up to 2.6 at the third PD with new lesions. We evaluated the time to the development of further metastases beyond those initially present. The median time to a first PD with new metastases was about 1 year. Interestingly enough, the time interval between a first and second as well as a second and third PD with new metastases was quite comparable.

Our findings are largely consistent with and extend those from prior epidemiological studies reporting on the incidence of specific synchronous $[2,6,12]$ and metachronous metastases [5]. In line with these reports, the majority of metachronous metastases was diagnosed 1.5 years after the primary tumor $[4,5]$. Minor discrepancies are evident in only one population-based study, which reports a higher frequency for metachronous hepatic and pulmonary metastases [4]. However, the liver and the lungs are consistently stated as the most frequent organs to be involved in mCRC.

To a certain extent, this observation may be driven by anatomy as the majority of colorectal cancers drain to the portal vein, thus promoting hematogenous spread to the liver [13]. Supporting this concept, the frequency of pulmonary metastases was increased in 
Fig. 6. Mean number of metastatic sites over the course of disease in patients with metastatic colorectal cancer. Mean numbers and standard deviations are displayed. PD = Progressive disease.

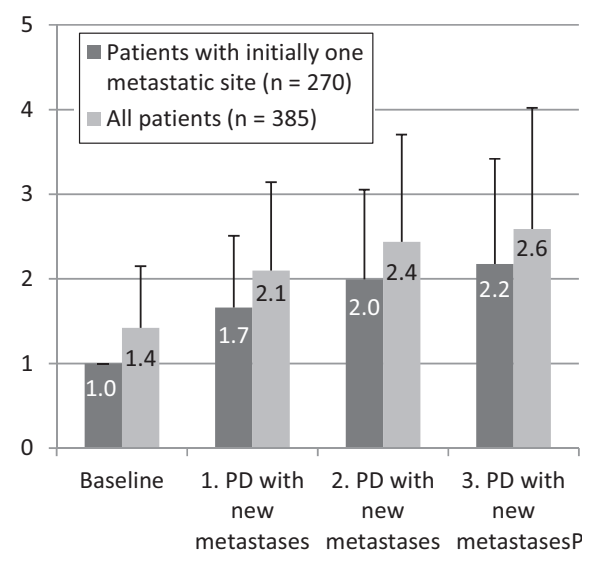

patients of our cohort with rectal cancer. Here, tumors partly drain through the inferior rectal vein to the vena cava, potentially promoting hematogenous dissemination in the lungs [14].

However, the anatomical localization of the primary tumor does not fully capture the occurrence of different metastatic patterns in mCRC. Further factors are likely to contribute to the site-specific appearance of metastases [13]. For example, certain alterations of the tumor cells appear to be associated with metastases to the lungs and lymph nodes $[15,16]$. Furthermore, a plethora of studies illustrates that specific metastatic patterns might reflect individual tumor biologies [8, 17-19]. For example, mutations in the KRAS gene were associated with metastases to the lungs and brain and less frequently to the liver $[8,18]$. Moreover, an activating BRAF mutation was associated with increased peritoneal involvement, distant lymph node metastasis, and decreased incidence of liver-limited disease [8].

Details on tumor genotype were limited in our patient cohort. However, the preponderance of peritoneal and distant lymph node metastases in colon cancer reflects the previous findings as $B R A F$ mutations are predominantly found in the right colon [20]. In addition, a differential metastatic pattern of synchronous and metachronous metastases underlines the notion of tumor biology as a key driver of clinical presentation [21].

The majority of patients in our cohort were initially diagnosed with metastases confined to one organ, i.e. primarily the liver. This subgroup is associated with a superior prognosis, which might be due to the option of metastectomy with curative intent [10, 22]. Of note, the current UICC classification and the American Joint Committee of Cancer (AJCC) differentiates between patients with oligometastatic disease (M1a) and those with disseminated disease (M1b)
[9]. In this context, M1a refers to patients with metastases confined to one organ, whereas M1b refers to patients with more than one organ involved or peritoneal carcinomatosis. However, some authors suggest to improve the prognostic accuracy by including the information on resectability into the staging system [23].

It is noteworthy that patients with initially one metastatic site maintained a reduced number of affected sites throughout the course of disease. This might reflect a more favorable tumor biology of initially oligometastatic disease, which contributes to the superior prognosis apart from an increased chance of resectability [22].

We acknowledge several limitations of the present investigation. As we have performed a retrospective analysis of a patient cohort treated at a specialized unit, selection bias cannot be excluded. To this end, such an approach cannot substitute epidemiological data. However, the presented frequencies of individual metastases are well in line with previous epidemiological studies. Hence, our findings may extend the limited data on organ-specific pattern and dynamic aspects of colorectal cancer metastases. Furthermore, details on tumor genotype were limited in our patient cohort (e.g. for RAS and BRAF mutation status), preventing a correlative analysis with specific metastatic patterns. Finally, we acknowledge the limited sensitivity and specificity of radiological imaging for the detection of colorectal cancer metastases with regard to lymph node and peritoneal metastases $[24,25]$. However, previous reports also based the diagnosis of colorectal cancer metastases to a major extent on radiological imaging results, as these are most widely available.

\section{Conclusion}

Taken together, our study analyzed the detailed localization of colorectal cancer metastases with regard to dynamic aspects. Our data illustrate that the tumor biology might be a key driver of clinical behavior. Furthermore, the development of new metastases over time appears to be constant during the course of disease, leading to an increase of metastatic sites over time. Our findings might stimulate further research to investigate the implications of localization and dynamics of colorectal cancer metastases on patient management.

\section{Disclosure Statement}

The authors declare no conflict of interest. Julian Holch received honoraria from Roche.

\section{References}

1 Robert Koch-Institut, Gesellschaft der epidemiologischen Krebsregister in Deutschland e.V. (eds): Krebs in Deutschland 2011/2012, ed 10. Berlin, 2015.
2 van der Geest LG, Lam-Boer J, Koopman M, Verhoef C, Elferink MA, de Wilt JH: Nationwide trends in incidence, treatment and survival of colorectal cancer patients with synchronous metastases. Clin Exp Metastasis $2015 ; 32: 457-465$.
3 van der Pool AE, Damhuis RA, Ijzermans JN, de Wilt JH, Eggermont AM, Kranse R, Verhoef C: Trends in incidence, treatment and survival of patients with stage IV colorectal cancer: a population-based series. Colorectal Dis 2012;14:56-61. 
4 Elferink MA, de Jong KP, Klaase JM, Siemerink EJ, de Wilt JH: Metachronous metastases from colorectal cancer: a population-based study in North-East Netherlands. Int J Colorectal Dis 2015;30:205-212.

5 Manfredi S, Bouvier AM, Lepage C, Hatem C, Dancourt V, Faivre J: Incidence and patterns of recurrence after resection for cure of colonic cancer in a well defined population. Br J Surg 2006;93:1115-1122.

6 Manfredi S, Lepage C, Hatem C, Coatmeur O, Faivre J, Bouvier AM: Epidemiology and management of liver metastases from colorectal cancer. Ann Surg 2006;244: 254-259.

7 Ghiringhelli F, Hennequin A, Drouillard A, Lepage C, Faivre J, Bouvier AM: Epidemiology and prognosis of synchronous and metachronous colon cancer metastases: a French population-based study. Dig Liver Dis 2014;46:854-858.

8 Lipsyc M, Yaeger R: Impact of somatic mutations on patterns of metastasis in colorectal cancer. J Gastrointest Oncol 2015;6:645-649.

9 Merkel S, Weber K, Croner RS, Golcher H, Gohl J, Agaimy A, Semrau S, Siebler J, Wein A, Hohenberger W, Wittekind C: Distant metastases in colorectal carcinoma: a proposal for a new M1 subclassification. Eur J Surg Oncol 2016;42:1337-1342.

10 Kohne CH, Cunningham D, Di Costanzo F, Glimelius B, Blijham G, Aranda E, Scheithauer W, Rougier P, Palmer M, Wils J, Baron B, Pignatti F, Schoffski P, Micheel S, Hecker H: Clinical determinants of survival in patients with 5-fluorouracil-based treatment for metastatic colorectal cancer: results of a multivariate analysis of 3825 patients. Ann Oncol 2002;13:308-317.

11 Sorbye H, Kohne CH, Sargent DJ, Glimelius B: Patien characteristics and stratification in medical treatment studies for metastatic colorectal cancer: a proposal for standardization of patient characteristic reporting and stratification. Ann Oncol 2007;18:1666-1672.
12 Mitry E, Guiu B, Cosconea S, Jooste V, Faivre J, Bouvier AM: Epidemiology, management and prognosis of colorectal cancer with lung metastases: a 30-year population-based study. Gut 2010;59:1383-1388.

13 Russo AL, Borger DR, Szymonifka J, Ryan DP, Wo JY, Blaszkowsky LS, Kwak EL, Allen JN, Wadlow RC, Zhu AX, Murphy JE, Faris JE, Dias-Santagata D, Haigis KM, Ellisen LW, Iafrate AJ, Hong TS: Mutational analysis and clinical correlation of metastatic colorectal cancer. Cancer 2014;120:1482-1490.

14 Hong TS, Clark JW, Haigis KM: Cancers of the colon and rectum: identical or fraternal twins? Cancer Discov 2012;2:117-121.

15 Urosevic J, Garcia-Albeniz X, Planet E, Real S, Cespedes MV, Guiu M, Fernandez E, Bellmunt A, Gawrzak S, Pavlovic M, Mangues R, Dolado I, Barriga FM, Nadal C, Kemeny N, Batlle E, Nebreda AR, Gomis RR: Colon cancer cells colonize the lung from established liver metastases through p38 MAPK signalling and PTHLH. Nat Cell Biol 2014;16:685-694.

16 Guo Q, Zhao Y, Chen J, Hu J, Wang S, Zhang D, Sun Y: BRAF-activated long non-coding RNA contributes to colorectal cancer migration by inducing epithelialmesenchymal transition. Oncol Lett 2014;8:869-875.

17 Tran B, Kopetz S, Tie J, Gibbs P, Jiang ZQ, Lieu CH, Agarwal A, Maru DM, Sieber O, Desai J: Impact of BRAF mutation and microsatellite instability on the pattern of metastatic spread and prognosis in metastatic colorectal cancer. Cancer 2011;117:4623-4632.

18 Kim MJ, Lee HS, Kim JH, Kim YJ, Kwon JH, Lee JO, Bang SM, Park KU, Kim DW, Kang SB, Kim JS, Lee JS, Lee KW: Different metastatic pattern according to the KRAS mutational status and site-specific discordance of KRAS status in patients with colorectal cancer. BMC Cancer 2012;12:347.
19 Gayyed MF, Abd El-Maqsoud NM, El-Hameed ElHeeny AA, Mohammed MF: c-MET expression in colorectal adenomas and primary carcinomas with its corresponding metastases. J Gastrointest Oncol 2015; 6:618-627.

20 Missiaglia E, Jacobs B, D’Ario G, Di Narzo AF, Soneson C, Budinska E, Popovici V, Vecchione L, Gerster S, Yan P, Roth AD, Klingbiel D, Bosman FT, Delorenzi M, Tejpar S: Distal and proximal colon cancers differ in terms of molecular, pathological, and clinical features. Ann Oncol 2014;25:1995-2001.

21 Slesser AA, Georgiou P, Brown G, Mudan S, Goldin R, Tekkis $P$ : The tumour biology of synchronous and metachronous colorectal liver metastases: a systematic review. Clin Exp Metastasis 2013;30:457-470.

22 Kohne CH, Poston G, Folprecht G, Ciardiello F, Ronga P, Beier F, Van Cutsem E: FOLFIRI plus cetuximab in patients with liver-limited or non-liver-limited RAS wild-type metastatic colorectal cancer: a retrospective subgroup analysis of the CRYSTAL study. Eur J Surg Oncol 2016;42:1540-1547.

23 Kennecke H, Yu J, Gill S, Cheung WY, Blanke CD Speers C, Woods R: Effect of Mla and M1b category in metastatic colorectal cancer. Oncologist 2014;19:720726

24 Choi HJ, Ju W, Myung SK, Kim Y: Diagnostic performance of computer tomography, magnetic resonance imaging, and positron emission tomography or positron emission tomography/computer tomography for detection of metastatic lymph nodes in patients with cervical cancer: meta-analysis. Cancer Sci 2010;101: 1471-1479.

25 Gonzalez-Moreno S, Gonzalez-Bayon L, Ortega-Perez G, Gonzalez-Hernando C: Imaging of peritoneal carcinomatosis. Cancer J 2009;15:184-189. 